\title{
Exciton effects in soliton and bipolaron lattice states of doped electron-phonon Peierls systems
}

\author{
(Running head: Exciton effects in doped Peierls systems)
}

Kikuo Harigaya Yukihiro Shimoi, and Shuji Abe

\author{
Fundamental Physics Section, \\ Electrotechnical Laboratory, \\ Umezono 1-1-4, Tsukuba, Ibaraki 305, Japan
}

(Received )

\begin{abstract}
Exciton effects on soliton and bipolaron lattice states are investigated using an electron-lattice Peierls model with long-range Coulomb interactions. The Hartree-Fock (HF) approximation and the single-excitation configurationinteraction (single-CI) method are used to obtain optical absorption spectra. We discuss the following properties: (1) The attraction between the excited electron and the remaining hole makes the excitation energy smaller when the correlations are taken into account by the single-CI. The oscillator strengths of the lower excited states become relatively larger than in the HF calculations. (2) We look at variations of relative oscillator strengths of two or three kinds of
\end{abstract}

*Electronic mail address: harigaya@etl.go.jp. 
excitons described by the single-CI. While the excess-electron concentration is small, the ratio of the oscillator strengths of the exciton with the lowest energy increases almost linearly. The oscillator strengths accumulate at this exciton as the concentration increases.

PACS numbers: 7135, 7840, 7138 


\section{Introduction}

It has been well known that correlation effects are present among $\pi$-electrons in conjugated polymers. For example:

- The envelope of the wavefunction of the midgap state of the neutral (spin) soliton in trans-polyacetylene has sites with spin density of the opposite sign [1]. This fact of the "negative spin density" has been explained by considering Coulomb interactions in the Su-Schrieffer-Heeger (SSH) model [2] of conjugated polymers [3].

- The electron paramagnetic resonance experiment of pernigraniline base [4], reported recently, has studied the spin distribution around a neutral soliton. It has been suggested that sites with negative spin density exist as the consequence of correlation effects.

- Nonlinear optical response functions of polydiacetylenes exhibit excitation structures owing to the presence of excitons. Their structures have been explained theoretically by using the intermediate exciton formalism [5].

Therefore, it is generally interesting to study Coulomb interaction effects in conjugated polymers. In fact, this problem has been considered by various authors for more than a decade since the SSH model [2] was proposed.

Electronic excitation structures in the half-filled conjugated polymers with the constant dimerization have been theoretically investigated by using the exciton formalism [5] and the exact diagonalization method [6], and also by

solving the time-dependent Hartree-Fock (HF) equations [7]. This problem 
was pointed out earlier, but excitation structures have been considered intensively only recently, relating with origins of nonlinear optical spectra [6-8]. The most remarkable consequence of correlation effects is that the lowest energy excitation has the largest oscillator strength. It is clearly seen when the optical spectra calculated by using the HF wavefunctions are compared with the spectra with the correlation effects. This fact does not depend on whether the higher correlations are taken into account by the single-excitation configuration-interaction (single-CI) method [5], or by the time-dependent HF formalism [7].

It is widely known that the soliton, polaron, and bipolaron lattices are present [9], when the SSH model [2], its continuum version, and the extended model with the term of the nondegeneracy [10] are doped with electrons or holes. New bands related with nonlinear excitations develop in the Peierls gap as the doping proceeds. When correlation effects are considered by the singleCI, the excitation structures exhibit the presence of excitons. There is one kind of exciton in the half-filled system, where the excited electron (hole) sits at the bottom of the conduction band (top of the valence band). We will call this as the "intercontinuum exciton". In the soliton lattice states of the doped SSH model for degenerate conjugated polymers, there are small gaps between the soliton band and the continuum states, i.e., valence and conduction bands. Therefore, the number of the kind of excitons would increase and their presence will be reflected in structures of the optical spectra. A new exciton, namely, "soliton-continuum exciton" will appear when the electron-hole excitation is considered between the soliton and one of the continuum bands. The main purpose of this paper is to embody the above picture. There have been a lot 
of investigations of correlation effects in doped SSH systems, but the work in the view point of excitons has been rarely performed. General properties of exciton effects on soliton lattice systems are the first interest of the paper. The portion of the spectral weights of the two kinds of excitons will be calculated and discussed.

There are two midgap bands, when the nondegenerate conjugated polymers are doped and bipolaron lattice states are formed. The excitation spectra will become complicated due to the increase of number of kinds of excitons. The second part of this paper will be devoted to this problem, and the relative spectral weight of each exciton will be studied again.

This paper is composed as follows. In $\S 2$, the model is introduced and the numerical method is explained. Results for the soliton lattice system are reported in $\S 3$. Effects of the nondegeneracy are investigated in $\S 4$. The paper is summarized in the final section.

\section{Model}

The following hamiltonian is used to discuss excitonic effects in soliton and bipolaron lattice states of Peierls systems:

$$
H=H_{\mathrm{SSH}}+H_{\text {int }} .
$$

The first term $H_{\mathrm{SSH}}$ of Eq. (1) is the SSH model:

$$
\begin{aligned}
H_{\mathrm{SSH}} & =-\sum_{i, \sigma}\left[t-\alpha y_{i}+(-1)^{i} \delta_{0} t\right]\left(c_{i, \sigma}^{\dagger} c_{i+1, \sigma}+\text { h.c. }\right) \\
& +\frac{K}{2} \sum_{i} y_{i}^{2}
\end{aligned}
$$


where $t$ is the hopping integral of the system without the dimerization; $\alpha$ is the electron-phonon coupling constant which changes the hopping integral linearly with respect to the bond variable $y_{i} ; \delta_{0} t$ is the Brazovskii-Kirova $(\mathrm{BK})$ term which measures the degree of the nondegeneracy (it was originally introduced in the continuum model [10]); $c_{i, \sigma}$ is an annihilation operator of the $\pi$-electron at the site $i$ with spin $\sigma$; the sum is taken over all the lattice sites of the periodic chain; and the last term with the spring constant $K$ is the harmonic energy of the classical spring simulating the $\sigma$-bond effects. The second term of Eq. (1) is the long-range Coulomb interaction in the form of the Ohno potential:

$$
\begin{aligned}
H_{\mathrm{int}} & =U \sum_{i}\left(c_{i, \uparrow}^{\dagger} c_{i, \uparrow}-\frac{n_{\mathrm{el}}}{2}\right)\left(c_{i, \downarrow}^{\dagger} c_{i, \downarrow}-\frac{n_{\mathrm{el}}}{2}\right) \\
& +\sum_{i \neq j} W\left(r_{i, j}\right)\left(\sum_{\sigma} c_{i, \sigma}^{\dagger} c_{i, \sigma}-n_{\mathrm{el}}\right)\left(\sum_{\tau} c_{j, \tau}^{\dagger} c_{j, \tau}-n_{\mathrm{el}}\right),
\end{aligned}
$$

where $n_{\mathrm{el}}$ is the number of $\pi$-electrons per site, $r_{i, j}$ is the distance between the $i$ th and $j$ th sites, and

$$
W(r)=\frac{1}{\sqrt{(1 / U)^{2}+\left(r / r_{0} V\right)^{2}}}
$$

is the Ohno potential. The quantity $W(0)=U$ is the strength of the onsite interaction, and $V$ means the strength of the long range part.

The model is treated by the HF approximation and the single-CI for the Coulomb potential. The adiabatic approximation is forced on the bond variables. The HF order parameters and bond variables are determined selfconsistently using the standard iteration method [11]. After the HF approximation $H \Rightarrow H_{\mathrm{HF}}$, we divide the total hamiltonian as $H=H_{\mathrm{HF}}+H^{\prime}$. The term $H^{\prime}$ becomes

$$
H^{\prime}=U \sum_{i}\left(c_{i, \uparrow}^{\dagger} c_{i, \uparrow}-\rho_{i, \uparrow}\right)\left(c_{i, \downarrow}^{\dagger} c_{i, \downarrow}-\rho_{i, \downarrow}\right)
$$




$$
\begin{aligned}
& +\sum_{(i, j), i \neq j} W\left(r_{i, j}\right)\left[\sum_{\sigma, \tau}\left(c_{i, \sigma}^{\dagger} c_{i, \sigma}-\rho_{i, \sigma}\right)\left(c_{j, \tau}^{\dagger} c_{j, \tau}-\rho_{j, \tau}\right)\right. \\
& \left.+\sum_{\sigma}\left(\tau_{i, j, \sigma} c_{j, \sigma}^{\dagger} c_{i, \sigma}+\tau_{j, i, \sigma} c_{i, \sigma}^{\dagger} c_{j, \sigma}-\tau_{i, j, \sigma} \tau_{j, i, \sigma}\right)\right]
\end{aligned}
$$

where $\rho_{i, \sigma}=\left\langle c_{i, \sigma}^{\dagger} c_{i, \sigma}\right\rangle$ and $\tau_{i, j, \sigma}=\left\langle c_{i, \sigma}^{\dagger} c_{j, \sigma}\right\rangle$ are Hartree-Fock order parameters. When we write the Hartree-Fock ground state $|g\rangle=\prod_{\lambda \text { :occupied }} c_{\lambda, \uparrow}^{\dagger} c_{\lambda, \downarrow}^{\dagger}|0\rangle$ and the single electron-hole excitations $|\mu \lambda\rangle=c_{\mu, \sigma}^{\dagger} c_{\lambda, \tau}|g\rangle$ ( $\mu$ means an unoccupied state; we assume both singlet and triplet excitations in this abbreviated notation), the matrix elements of the HF part and the excitation hamiltonian become as follows:

$$
\begin{aligned}
\left\langle\mu^{\prime} \lambda^{\prime}\left|\left(H_{\mathrm{HF}}-\left\langle H_{\mathrm{HF}}\right\rangle\right)\right| \mu \lambda\right\rangle & =\delta_{\mu^{\prime}, \mu} \delta_{\lambda^{\prime}, \lambda}\left(E_{\mu}-E_{\lambda}\right), \\
\left\langle\mu^{\prime} \lambda^{\prime}\left|\left(H^{\prime}-\left\langle H^{\prime}\right\rangle\right)\right| \mu \lambda\right\rangle & =2 J \delta_{S}-K,
\end{aligned}
$$

where $E_{\mu}$ is the energy of the HF orbital, $\delta_{S}=1$ for spin singlet, $\delta_{S}=0$ for spin triplet, and

$$
\begin{aligned}
J\left(\mu^{\prime}, \lambda^{\prime} ; \mu, \lambda\right) & =\sum_{i, j} V_{i, j}\left\langle\mu^{\prime} \mid i\right\rangle\left\langle\lambda^{\prime} \mid i\right\rangle\langle j \mid \mu\rangle\langle j \mid \lambda\rangle \\
K\left(\mu^{\prime}, \lambda^{\prime} ; \mu, \lambda\right) & =\sum_{i, j} V_{i, j}\left\langle\mu^{\prime} \mid j\right\rangle\left\langle\lambda^{\prime} \mid i\right\rangle\langle j \mid \mu\rangle\langle i \mid \lambda\rangle
\end{aligned}
$$

with $V_{i, i}=U, V_{i, j}=W\left(r_{i, j}\right)$ for $i \neq j$. The diagonalization of the total hamiltonian $H$ gives the set of the excited states $\{|\kappa\rangle\}$ within the single-CI method. In the actual calculation, we limit the spin configurations to the singlet excitations which are the main interests of optical excitations.

We assume a geometry of a ring for a polymer chain, in order to remove edge effects. If we use an open boundary, the dimerization and thus the Peierls gap becomes larger near the two edges, and this might result in artifacts of optical spectra. We shall use the coordinate of $j$ th carbon atoms,

$$
\left(r \cos \frac{2 \pi j}{N}, r \sin \frac{2 \pi j}{N}, 0\right)
$$


where $r=N a /(2 \pi)$ is the radius of the polymer ring; $N$ is the system size and $a$ is the lattice constant. The electric field of light is parallel to the $x-y$ plane. In order to obtain optical spectra which are independent of the relative positions of solitons with respect to the direction of light, we shall sum up two spectra where light is along with the $x$ - and $y$-directions. So, we use the following formula of the spectrum:

$$
\sum_{\kappa} E_{\kappa} P\left(\omega-E_{\kappa}\right)(\langle g|x| \kappa\rangle\langle\kappa|x| g\rangle+\langle g|y| \kappa\rangle\langle\kappa|y| g\rangle) .
$$

Here, $P(\omega)=\gamma /\left[\pi\left(\omega^{2}+\gamma^{2}\right)\right]$ is the Lorentzian distribution $(\gamma$ is the width), $E_{\kappa}$ is the electron-hole excitation energy, and $|g\rangle$ means the ground state. In eq. (11), the quantity,

$$
E_{\kappa}(\langle g|x| \kappa\rangle\langle\kappa|x| g\rangle+\langle g|y| \kappa\rangle\langle\kappa|y| g\rangle)
$$

is the oscillator strength of the excited state $|\kappa\rangle$. Applying electric field to the ring-shaped polymer simulates the situation that polymer chains are oriented randomly in every direction within the $x-y$ plane. The average over orientations are effectively performed. The similar idea has been used in the recent paper by Abe et al [5].

The system size is chosen as $N=80,100,120$ when the electron number is even (it is varied from $N_{\mathrm{el}}=N, N+2, N+4, N+6$ to $N+8$ ), because the size around 100 is known to give well the energy gap value of the infinite chain. More larger system size becomes tedious for doing single-CI calculations which call for huge computer memories. When there is one soliton, we take $N=81,101,121$, and use the periodic boundary condition also.

In principle, we have to adjust parameters and find appropriate ones in order to reproduce experimental data, such as, the energy gap and the dimer- 
ization amplitude. But, we will change parameters arbitrary in a reasonable range in order to look at excitonic effects clearly. The Coulomb parameters are changed within $0 \leq V \leq U \leq 5 t$, and we show results for $U=2 V=2 t$ and $=4 t$ as the representative cases. Other parameters, $t=1.8 \mathrm{eV}, K=21 \mathrm{eV} / \AA^{2}$,

and $\alpha=4.1 \mathrm{eV} / \AA$, are fixed in view of the general interests of this paper. All the quantities of energy dimension are shown in the units of $t$.

\section{Soliton lattice systems}

Figure 1 shows the typical lattice configuration and excess-electron density distribution for $N=100, N_{\mathrm{el}}=104, U=4 t, V=2 t$, and $\delta_{0}=0$. Both quantities are the smoothed data after the removal of small oscillations between even- and odd-number sites. There are four charged solitons due to the excesselectron number $N_{\mathrm{el}}-N=4$. Solitons are arrayed equidistantly. The excesselectron density shows the oscillation of the charge density with its maxima at the soliton centers.

Next, let us look at optical spectra and consider exciton effects. We calculate also the optical spectra from single-electron excitations among HF energy levels as references of exciton effects. The typical optical spectra within the HF approximation and with the single-CI are shown as Figs. 2 and 3, respectively. The broadening $\gamma=0.05 t$ is used. The Coulomb parameters are $U=4 t$ and $V=2 t$. Relatively strong Coulomb interactions are taken in order to look at the exciton effects in the optical response clearly. The system size and the electron number are $\left(N, N_{\mathrm{el}}\right)=(101,102),(100,102),(100,104)$, for (a), (b), and (c) (of Figs. 2 and 3), respectively. 
In order to characterize properties of optical excitations, in other words, to identify whether the excitons are formed between valence and conduction bands ("intercontinuum exciton"), or between soliton and conduction bands ("soliton-continuum exciton"), we calculate the component of the electron-hole excitation between the soliton and conduction bands for the absorption spectra within $\mathrm{HF}$ as well as those with the single-CI. Results are shown in Figs. 2 and 3 , superposed with the optical spectra. We can easily determine the position of the optical gap by comparing components of each kind of exciton. The optical gap of one exciton is located at the lowest energy where the component is larger than that of the other exciton.

As we proceed from Fig. 2(a) to Fig. 2(c) with increasing the soliton concentration, the contribution from the transition between the soliton band and the valence band becomes larger than that between the soliton and conduction bands. In other words, the optical transition between the soliton and conduction bands rapidly develops as the soliton concentration increases. The energy positions of optical gaps of excitons are shown by the triangles at the top of each figure. The lowest optical gap is about $0.7 t, 0.9 t$, and $1.0 t$ in Figs. 2(a), (b), and (c), respectively, and is slightly increasing. The almost constant behavior can be explained as follows. The HF order parameter $\tau_{i, j, \sigma}$ introduces an additional bond order, and thus increases the energy gap. In contrast, the lowest optical gap is a decreasing function of the soliton concentration in the free-electron case. Therefore, the decrease of the optical gap in the free electron case is suppressed by the increase of the energy gap in the presence of long-range Coulomb interactions. The optical gap of the transition between the continuum states is about $1.6 t, 1.7 t$, and $1.9 t$ for the three figures. This 
quantity becomes larger with the concentration, due to the increase of number of states in the soliton band.

We shall look at the optical spectra by HF and single-CI calculation in order to discuss exciton effects. They are shown in Fig. 3. The optical gap of the soliton-continuum exciton is $0.6 t, 0.7 t$, and $0.8 t$ in Figs. 3(a), (b), and (c), respectively. The optical gap of the intercontinuum exciton is 1.4t, 1.4t, and $1.6 t$ for the three figures. Here, we have regarded that the energy position of the lowest excitation where the component of the soliton-continuum exciton becomes smaller than 0.5 , is the optical gap of the intercontinuum exciton. Both optical gaps decrease apparently from those of Fig. 2. This is due to the binding of an electron and a hole in the CI treatment. We also find that the soliton-continuum exciton has the larger total oscillator strength than that of Fig. 2. This is due to the one dimensionality, discussed in ref. [5].

There are many small structures in the optical spectra due to the finite system size. They could be removed by doing calculations for larger systems, as has been done in the paper [12] with reducing the dimension of the matrix of CI excitations by means of the translational symmetry for the half-filled hamiltonian. But, the reduction of the system size is difficult for soliton lattice states owing to the periodicity of the system which changes with the soliton concentration. In order to pursue the change of optical excitation characters systematically for various combinations of parameters, we rather perform calculations for small system sizes around 100 and analyze numerical data as functions of the soliton concentration. In fact, when the optical gap and the ratio of the total oscillator strengths of the soliton-continuum exciton are plotted against the soliton concentration, $\left(N_{\mathrm{el}}-N\right) / N$, the plots are arrayed rather 
smoothly. We shall look at the data in Figs. 4 and 5.

Figure 4 summarizes the optical gaps of the two kinds of excitons. They are calculated by the HF followed by the single-CI. Figures 4(a) and (b) are for $U=$ $2 V=2 t$ and $=4 t$, respectively. The gaps of the intercontinuum exciton and soliton-continuum exciton are shown by closed and open squares, respectively. The optical gap of the soliton-continuum exciton is almost independent of the concentration, owing to the balance between the decreasing due to the soliton concentration change and the widening of the energy gap owing to the presence of long-range Coulomb interactions. The gap of the intercontinuum exciton increases rapidly when the concentration is larger than 2.5 percent. This is due to the fact that the number of states in the soliton band increases, and thus the energy gap between continuum states increases. It seems that these properties are common for the two Coulomb parameter sets. The optical gaps are larger for stronger Coulomb interactions. This is due to the larger bond order parameters which enhance the Peierls gap of the system.

Figure 5 shows the ratio of the oscillator strengths of the soliton-continuum exciton, plotted against the soliton concentration. The closed and open squares are the results of the HF-CI and HF calculations, respectively. The closed squares have the larger ratio than the open ones. This is one of exciton effects. When the concentration is near zero, the ratio varies almost linearly. This would be the natural consequence for low concentrations, because interactions among solitons are exponentially small and thus the ratio is proportional to the number of solitons. The increase near the zero concentration is slower for the stronger $U$ and $V$ of Fig. 5(b) than in Fig. 5(a). This would be due to that the soliton width is smaller for the stronger Coulomb repulsions and the 
portion of regions with nearly perfect dimerization strengths is larger. The increase of the ratio saturates at about 5 percent in Fig. 5(a) and at about 7 percent in Fig. 5(b). The soliton-continuum exciton becomes like a free exciton at larger concentrations owing to the formation of the soliton band.

\section{Bipolaron lattice systems: Confinement ef- fects}

There exist only two kinds of degenerate conjugated polymers. They are transpolyacetylene and pernigraniline base. All the other conjugated polymers have nondegenerate ground states. Therefore, it is also interesting to look at exciton effects in this type of polymers. The structures of nondegenerate conjugated polymers are generally complex, including aromatic rings and side chains. By this reason, the simple SSH-type models directly simulate only the structures of linear-chain polymers: trans- and cis-polyacetylenes. However, the SSH model with the non-zero BK term has the nondegenerate ground state which is one of the general properties described by the simple SSH-type models. Therefore, it is of general interests to look at excitonic effects on nondegenerate conjugated polymers. We shall consider the model Eq. (1) with $\delta_{0}=0.02$. This small BK term gives rise to the large energy difference between the ground state and the excited state with the reversed phase of the bond alternation pattern, after calculations of adiabatic approximation with the full lattice relaxation. The Coulomb and lattice parameters are the same as in the previous section.

Figure 6 shows the static lattice configuration and the excess-electron den-

sity distribution for $N=100, N_{\mathrm{el}}=104, U=4 t$, and $V=2 t$. Figure 6 (a) 
displays the lattice configuration. The ground state with the positive bond variable is more stable than the state with the negative bond variable. Therefore, the region with the negative bond variable becomes smaller than in Fig. 1(a), and two neighboring solitons come closer each other to form a bipolaron. There are two bipolarons in Fig. 6(a). The electron distribution pattern in Fig. 6(b) reflects the fact that two solitons are confined to form a bipolaron. If the confinement is more stronger, two peaks in the charge density distribution change into a single peak.

As we have done in the previous section, we shall calculate optical absorption spectra by using the HF wavefunctions only as well as by performing single-CI calculations. Figure 7 shows the results of HF absorption (thin lines) and those of HF plus single-CI absorption (thick lines). The following parameters are the same as in Fig. 6: $U=2 V=4 t$ and $N=100$. The electron number is $N_{\mathrm{el}}=102$ for Fig. $7(\mathrm{a})$ and $N_{\mathrm{el}}=104$ for Fig. $7(\mathrm{~b})$. The broadening $\gamma=0.05 t$ is used. The major difference between the HF and HF-CI absorption is that the overall feature in the HF absorption shifts to lower energies in the HF-CI one. This is one of the exciton effects.

A single bipolaron has two midgap states. Then, there are two midgap bands in the bipolaron lattice system. The number of excitons is three. We shall call them as follows: the photo-excited state from the upper bipolaron band to the conduction band as the "lower bipolaron-continuum exciton", the exciton from the lower bipolaron band to the conduction band as the "upper bipolaron-continuum exciton", and the exciton between the continuum states as the "intercontinuum exciton". In Fig. 7(a), the optical gaps of the lower bipolaron-continuum exciton, the upper bipolaron-continuum exciton, and the 
inter-continuum exciton are about $0.5 t, 0.7 t$, and $1.6 t$, respectively. In Fig. $7(\mathrm{~b})$, they are about $0.6 t, 0.9 t$, and $1.6 t$, respectively. The total oscillator strength of the upper bipolaron-continuum exciton is always much smaller than that of the other two excitons. This property is already seen in the free electron case and is independent of the magnitudes of $U$ and $V$. As the concentration of the bipolarons increases, the oscillator strength of the lower bipolaron-continuum exciton enhances rapidly, and that of the intercontinuum exciton becomes smaller.

In order to analyze the concentration dependences systematically, we perform calculations for several combinations of the system size $N$ and the electron number $N_{\mathrm{el}}$. The electron number is always even, and then the bipolaron number is half of the excess-electron number $N_{\mathrm{el}}-N$. Figure 8 summarizes the optical gaps of three kinds of excitons (single-CI description), plotted against the excess-electron concentration: $\left(N_{\mathrm{el}}-N\right) / N$. We take two combinations of Coulomb parameters: $(U, V)=(2 t, 1 t)$ [Fig. 8(a)] and $(4 t, 2 t)$ [Fig. 8(b)]. The optical gaps of the intercontinuum exciton, and upper (lower) bipolaroncontinuum excitons are shown by the filled, and crossed (open) squares, respectively. The arrays of the plots behave smoothly, so it seems that the size effects are small. The optical gaps of the intercontinuum exciton and upper bipolaroncontinuum exciton increase gradually as functions of the excess-electron concentration. But, the increase of the lowest optical gap is suppressed, and it is nearly constant for concentrations larger than about $5 \%$. The similar behaviors have been seen in Fig. 3 for the soliton lattice case.

Finally, we consider character of optical excitations by looking at the ratios of the total oscillator strengths of lower and upper bipolaron-continuum 
excitons. Figure 9 shows the results plotted against the excess-electron concentration. The squares are the data of the lower bipolaron-continuum excitons, and circles are the data of the upper ones. The open symbols are the data of the HF absorption, and the closed ones are for the HF-CI calculations. The ratios in the HF-CI absorption spectra are calculated as we have done in the soliton lattice case. Here, we assign the optical excitation to the exciton whose component is the largest among three excitons. The closed squares have the larger ratio than the open ones, due to exciton effects. The increase near the zero concentration of the lower bipolaron-continuum exciton is suppressed for the stronger $U$ and $V$ of Fig. 9(b) than in Fig. 9(a). In other words, the increase of the ratio is steeper for weaker Coulomb interactions. This would be due to that the bipolaron width is smaller for the stronger Coulomb repulsions and the portion of regions with nearly perfect dimerization strengths is larger. The increase of the ratio nearly saturates at about 5 percent. The lower bipolaron-continuum exciton becomes like a free exciton at larger concentrations. In contrast, the ratio of the upper bipolaron-continuum exciton is always small, and it is smaller than about 0.20 . This smallness is already seen in the free electron case, and persists when there are Coulomb interactions. The same property in the optical spectra of the polaron and the bipolaron has been discussed in [13].

\section{Summary}

We have looked at exciton effects on soliton and bipolaron lattice states in a model of the interacting electron lattice system with long-range Coulomb 
interactions. The Hartree-Fock approximation and the single-CI method have been used to obtain optical absorption spectra. We have discussed the following properties:

(1) By comparison of the HF absorption with the HF-CI one, we have seen exciton effects which are similar to those, discussed for the half-filled systems in refs. $[5,7]$. The attraction between the excited electron and the remaining hole makes the excitation energy smaller when the correlations are taken into account by the single-CI. The oscillator strengths of the lower excited states become relatively larger than in the HF calculations.

(2) We have looked at variations of relative oscillator strengths of two or three kinds of excitons described by the single-CI. While the excess-electron concentration is small, the ratio of the oscillator strengths of the lowest exciton increases almost linearly. And, more than 80 percent of the oscillator strengths accumulate at the lowest exciton when the excess-electron concentration is larger than about 5 percent. It seems that this accumulation is very rapid as the concentration increases.

(3) The variations of the optical gaps and the ratios of the oscillator strengths as functions of the concentration of the nonlinear excitations (solitons or bipolarons) are rather smooth. It seems that how to systematically deal with such kinds of the numerical data obtained from numerical diagonalizations of finite systems is not fully investigated yet. We have looked variations as functions of the concentration, and have found the smooth variations. This fact was not stressed upon earlier, and plotting as a function of the concentration will be one of the useful methods for future investigations of systems with nonlinear excitations. 


\section{Acknowledgements}

The author acknowledges useful discussion with Prof. S. Stafström and Dr.

Akira Takahashi. 


\section{References}

[1] Kuroda S and Shirakawa H 1987 Phys. Rev. B 359380

[2] Su W P, Schrieffer J R and Heeger A J 1980 Phys. Rev. B 222099

[3] Yonemitsu K and Wada Y 1988 J. Phys. Soc. Jpn. 57 3875; Sasai M and Fukutome H 1983 Prog. Theor. Phys. 69373

[4] Long S M, Sun Y, MacDiarmid A G and Epstein A J 1994 Phys. Rev.

Lett. 723210

[5] Abe S, Yu J and Su W P 1992 Phys. Rev. B 458264

[6] Guo D, Mazumdar S, Dixit S N, Kajzar F, Jarka F, Kawabe Y and Peyghambarian N 1993 Phys. Rev. B 481433

[7] Takahashi A and Mukamel S 1994 J. Chem. Phys. 1002366

[8] Abe S, Schreiber M, Su W P and Yu J 1992 Phys. Rev. B 459432

[9] Horovitz B 1981 Phys. Rev. Lett. 46742

[10] Brazovskii S A and Kirova N N 1981 JETP Lett. 334

[11] Terai A and Ono Y 1986 J. Phys. Soc. Jpn. 55213

[12] Abe S, Schreiber M, Su W P and Yu J 1992 Mol. Cryst. Liq. Cryst. 217 1.

[13] Shimoi Y, Abe S and Harigaya K Mol. Cryst. Liq. Cryst. (to be published). 


\section{FIGURE CAPTIONS}

Fig. 1. (a) The dimerization order parameter, $(-1)^{n}\left(y_{n+1}-y_{n}\right) / 2$, and (b) the excess-electron density, $\left(\rho_{n-1}+2 \rho_{n}+\rho_{n+1}\right) / 4$. The parameters are $\delta=0$, $U=4 t, V=2 t, N=100$, and $N_{\mathrm{el}}=104$. See the text for the other parameters.

Fig. 2. The optical absorption spectra calculated with the HF wavefunctions for (a) $\left(N, N_{\text {el }}\right)=(101,102)$, (b) $(100,102)$, and (c) $(100,104)$. The broadening $\gamma=0.05 t$ is used. The units of the abscissa are arbitrary. The component of the electron-hole excitation between the soliton and conduction bands are shown by dots which are connected with thin lines. The energy positions of the optical gaps are shown by the triangles at the top of each figure.

Fig. 3. The optical absorption spectra calculated with the HF plus single-CI wavefunctions for (a) $\left(N, N_{\text {el }}\right)=(101,102)$, (b) $(100,102)$, and (c) $(100,104)$. The broadening $\gamma=0.05 t$ is used. The units of the abscissa are arbitrary. The component of the electron-hole excitation between the soliton and conduction bands are shown by dots which are connected with thin lines. The energy positions of the optical gaps are shown by the triangles at the top of each figure.

Fig. 4. The optical gaps in the single-CI of the "intercontinuum exciton" (filled squares) and of the "soliton-continuum exciton" (open squares), plotted against the soliton concentration. Coulomb interaction parameters are 
$(U, V)=(2 t, 1 t)$ for $(\mathrm{a})$, and $(4 t, 2 t)$ for $(\mathrm{b})$.

Fig. 5. The ratio of the total oscillator strength of the "soliton-continuum exciton" as a function of the soliton concentration. The open squares are the data for the HF absorption, while the filled ones for the HF-CI absorption. Coulomb interaction parameters are $(U, V)=(2 t, 1 t)$ for $(\mathrm{a})$, and $(4 t, 2 t)$ for (b).

Fig. 6. (a) The dimerization order parameter, $(-1)^{n}\left(y_{n+1}-y_{n}\right) / 2$, and (b) the excess-electron density, $\left(\rho_{n-1}+2 \rho_{n}+\rho_{n+1}\right) / 4$. The parameters are $\delta=0.02$, $U=4 t, V=2 t, N=100$, and $N_{\mathrm{el}}=104$. See the text for the other parameters.

Fig. 7. The optical absorption spectra calculated with the HF (thin lines) and HF-CI (full lines) wavefunctions for (a) $\left(N, N_{\mathrm{el}}\right)=(100,102)$ and (b) $(100,104)$. The broadening $\gamma=0.05 t$ is used. The units of the abscissa are arbitrary.

Fig. 8. The optical gaps in the single-CI of the "intercontinuum exciton" (filled squares), of the "upper bipolaron-continuum exciton" (crossed squares), and of the "lower bipolaron-continuum exciton" (open squares), plotted against the excess-electron concentration. Coulomb interaction parameters are $(U, V)=$ $(2 t, 1 t)$ for $(\mathrm{a})$, and $(4 t, 2 t)$ for $(\mathrm{b})$.

Fig. 9. The ratio of the total oscillator strength of the "upper (lower) 
bipolaron-continuum exciton" as functions of the excess-electron concentration. The squares are for the lower exciton, and the circles are for the upper exciton. The open symbols are the data for the HF absorption, while the filled ones for the HF-CI absorption. Coulomb interaction parameters are $(U, V)=(2 t, 1 t)$ for $(\mathrm{a})$, and $(4 t, 2 t)$ for $(\mathrm{b})$. 